Research Article

\title{
Finite Element Analysis of the Expression of Plantar Pressure Distribution in the Injury of the Lateral Ligament of the Ankle
}

\author{
Dian Wang ${ }^{1,2,3}$, Ping Cai ${ }^{1,2,3}$ \\ ${ }^{1}$ School of Electronic Information and Electrical Engineering, Shanghai Jiao Tong University, Shanghai 200240, China. \\ ${ }^{2}$ Shanghai Engineering Research Center for Intelligent Diagnosis and Treatment Instrument, Shanghai 200240, China. \\ ${ }^{3}$ Institute of Rehabilitation Engineering \& Technology, Shanghai 200240, China. \\ Corresponding authors. E-mail: dxcui@sjtu.edu.cn; sjie@sjtu.edu.cn
}

Received: Sep. 27, 2018; Accepted: Sep. 6, 2019; Published: Sep. 6, 2019.

Citation: Dian Wang, Ping Cai, Finite Element Analysis of the Expression of Plantar Pressure Distribution in the Injury of the Lateral Ligament of the Ankle. Nano Biomed. Eng., 2019, II (3): 290-296.

DOI: 10.5101/nbe.v11i3.p290-296.

\begin{abstract}
The program of treatment and rehabilitation of chronic ankle instability is different according to the impairment degree of lateral ligament of the ankle. The diagnosis methods of lateral ligament's injury are magnetic resonance imaging (MRI) and arthroscopy, which are either invasive or timeconsuming. This study aims to analyze the mapping relation between lateral ligaments' injury and center of pressure (COP) position shift via finite element analysis in order to explore a method that can diagnose lateral ligaments' injury. Two typical finite element method (FEM) bone models of heelstrike and toes-off phase are built according to the gait metrics. The lateral ligament is simulated by adding the spring to the model in the anatomical position and the injury of lateral ligament is simulated by reducing the stiffness of the spring. The contact pressure between the foot and support plate and COP coordinates are analyzed. The displacement of anterolateral shift of COP coordinates is mapped to the injury degree of lateral ligament. This study provides feasibility to diagnose lateral ligament injury by plantar pressure distribution.
\end{abstract}

Keywords: Mechanical ankle instability; Lateral ligament injury; Finite element method; Center of pressure

\section{Introduction}

Ankle is of the importance weight-bearing joint and plays a crucial role in keeping balance and posture control during standing and walking. The injury of ligament around the ankle joint can directly lead to lower mechanical stability of the ankle joint and repeated ankle sprains which would weaken the lateral ligaments further, worsen the equilibrium and increase the risk of complications like osteochondral lesions of the talus [1]. The Injury of lateral ligaments of the ankle locates in talofibulare anterius ligament, talofibulare posterius ligament and calcaneofibulare ligament [2]. The degree of the aforementioned ligaments is generally subdivided into three levels clinically. As the program of treatment or rehabilitation is developed according to the degree of the ligaments' injury. It is necessary to evaluate the degree of severity of the ligaments' injury.

The inspection methods of chronic ankle instability 
(CAI) includes anterior drawer test, talar tilt test, arthroscopy and magnetic resonance imaging (MRI) [3, 4]. The positive threshold of anterior drawer test and talar tilt test are subjective, arthroscopy and MRI are either invasive or time-consuming. This study makes effort exploring the possibility of diagnosis the injuries of lateral ligaments by plantar pressure distribution via finite element method. Plantar pressure distribution has been adopted by many researches to diagnose and classify the disease of foot and ankle. Keijsers et al. classified the patients with forefoot pains via the artificial neural network which was trained by the parameters of the plantar pressure distribution images [5]. Mickle et al., studied the likelihood of elder people falling with plantar peak pressure [6].

Finite element method plays an important role in the analysis of the expression of plantar pressure distribution. Niu et al. utilized finite element methods to analyze the interaction force of the foot arch in the support phase of the gait and got some useful conclusions on arch bone fractures [7]. Jia et al. analyzed the plantar pressure distribution of the subjects wearing high heeled shoes [8]. Cheung et al. assembled the bone model into the soft tissue model and studied the effect of the change of soft tissue stiffness on plantar distribution by finite element method [9]. However, the current studies only analyzed the expression of plantar pressure distribution of the neural position model, models of other gait phases, like heel-strike and toes-off phases, are not well studied. Sprains are more like to happen in heel-strike and toesoff phases, and this study will analyze plantar pressure distributions of those phases.

Many studies have utilized center of pressure (COP) parameters to analyze CAI patients. Nyska et al. placed six pressure sensing cells under heel, arch, metatarsals and toes to measure aforementioned anatomical regions [15]. Their result showed that CAI patients' peak pressure and COP trajectory of CAI differs from the normal. Morrison et al. found that lateral plantar pressure of CAI patients was statistically different from the normal when the subjects were jogging [16]. However, these studies were more concerned with the clinical features of CAI and did not go into studying the intrinsic reason that caused the change of such clinical features.

Without loss of generality, a typical biomechanical model of foot and ankle for finite element analysis is introduced in this study, and the model is used for the exploration of the change of plantar pressure distribution caused by the ligament injury and the possibility of diagnosis mechanical ankle instability via plantar pressure distribution.

\section{Experimental}

\section{Finite element model and material parameters}

The angles of the major joints of ankle and foot change continuously during walking and those angles can be modified in SolidWorks so that the corresponding joints are reassembled, presenting the model of a new gait phase. The modification of the joints' angles must be within the movement range of the joints to reflect the practical condition [18]. The heel-strike model was obtained by adjusting the angle of the joint of tibia and talus and the joint of fibula and talus in the neutral position model into dorsiflexion 15. And the toes-off model was obtained by adjusting the angle of the metatarsophalogeal joints in the neutral position model into plantar flexion 20. The soft tissue of foot and ankle was simulated by the cystic models which were built according to the appearance of foot and ankle in the heel-strike and toes-off phase respectively. The soft tissue model and the bone model were assembled together and imported into ANASY 18.0 workbench. The musculoskeletal ankle and foot model is showed in Fig. 1.

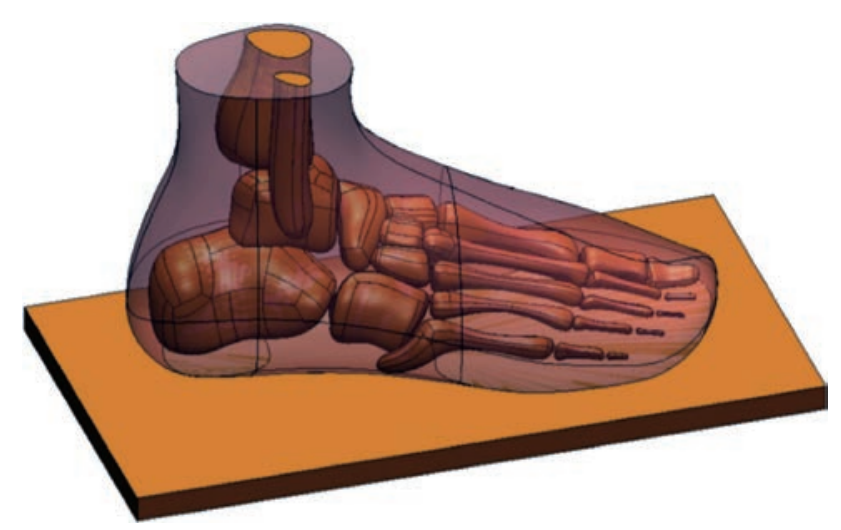

Fig. 1 Musculoskeletal ankle and foot model.

The model of foot and ankle was meshed with tetrahedron element of $6 \mathrm{~mm}$ size length. There were 99145 nodes and 52651 elements after the meshing. The face mesh was used for the support plate. The contact type of the joint of tibia and talus and the joint of fibula and talus was set to be frictional with the coefficient of 0.002 [10], which allowed the bones of the aforementioned two joints to have relatively 
displacement both in the normal and tangent directions. Other joints that have small range of motion such as interphalangeal joints, metatarsophalogeal joints and intertarsal joints were set to be bonded which could not move relative to each other. Metatarsophalangeal and interphalangeal joints have large range of motion, but they cannot move relatively during the specific phase of the gait; therefore, these joints were also set to be bonded [11]. The foot and ground interaction was set to be frictional with the frictional coefficient of 0.6 [12].

The springs were used in the finite element model according to the anatomical structural in order to simulate the three lateral ligaments: talofibulare anterius ligament, talofibulare posterius ligament and calcaneofibulare ligament, as well as the deltoid ligaments. The material properties are showed as Table 1 [13].

The top surface of tibia, fibula and soft tissue was fixed support and the displacement load was exerted

Table 1 Material properties of the finite element model

\begin{tabular}{ccc}
\hline Material & Young's modulus & Poisson's rate \\
\hline Bone & $7300 \mathrm{MPa}$ & 0.3 \\
Soft tissue & $0.45 \mathrm{MPa}$ & 0.49 \\
Support plate & $20000 \mathrm{MPa}$ & 0.1 \\
ATaF & $0.089 \mathrm{kN} / \mathrm{mm}$ & -- \\
PTaF & $0.775 \mathrm{kN} / \mathrm{mm}$ & -- \\
$\mathrm{CF}$ & $0.452 \mathrm{kN} / \mathrm{mm}$ & -- \\
PTaT & $0.821 \mathrm{kN} / \mathrm{mm}$ & -- \\
$\mathrm{CT}$ & $0.302 \mathrm{kN} / \mathrm{mm}$ & -- \\
TiN & $0.039 \mathrm{kN} / \mathrm{mm}$ & -- \\
\hline
\end{tabular}

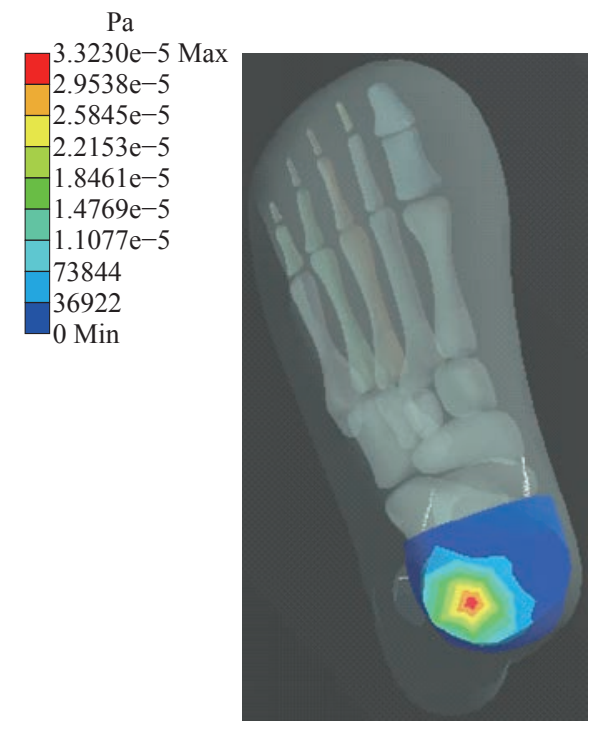

(a) beneath the support plate [14]. The contact pressure distribution between foot and the support plate was solved.

\section{Results and Discussion}

Fig. 2 presents the results of finite element models solution in this study. Toes-off and heel-strike phases were studied.

The results of heel-strike and toes-off were in accordance with results in the study of Morrison et al. and Tao et al., which were built by reconstructing MRI images. And these results can be used as a baseline when analyzing plantar pressure distribution of injured ligament model. Mechanical ankle instability patients injured their lateral ligaments and the injury degree of the ligament could be simulated through altering the stiffness of the spring in the model, which made it able to quantify the effect of injury of lateral ligament on plantar pressure distribution. Fig. 3 and 4 show the plantar pressure distributions when lateral ligaments in finite elements models of heel-strike and toes-off were half and complete injury.

The stiffness of springs simulating talofibulare anterius ligament and calcaneofibulare ligament was set to half of the normal stiffness in Fig. 3(a) and (c), and to zero in Fig. 3(b) and (d), respectively, to simulate the injury of the ligaments. In the toes-off gait phase, talofibulare posterius ligament was completely injured; and therefore it could be ignored. Fig. 3

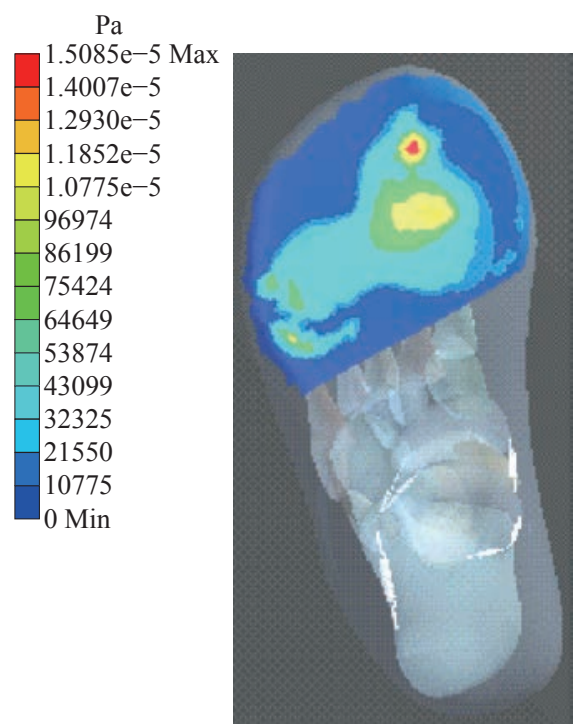

(b)

Fig. 2 Contact pressure distribution of toes-off and heel strike phase: (a) Plantar pressure of heel strike; (b) plantar pressure of toes off. 

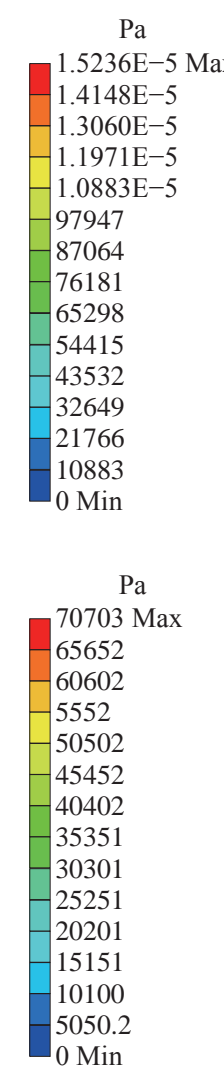

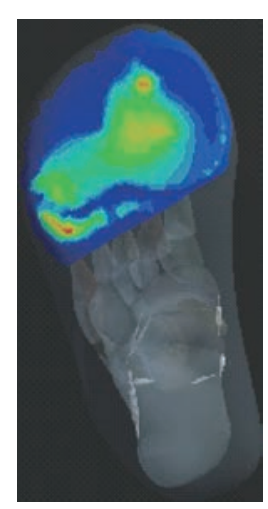

(a)

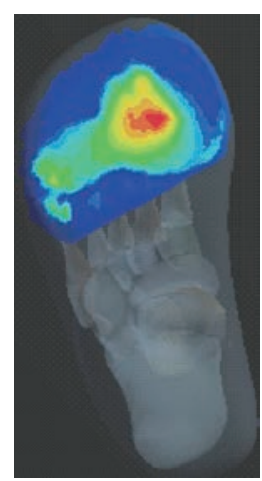

(c)

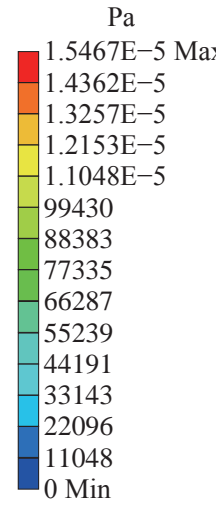

$\mathrm{Pa}$

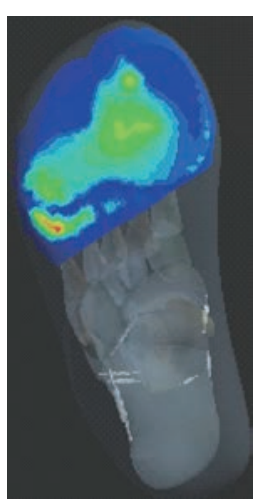

(b)

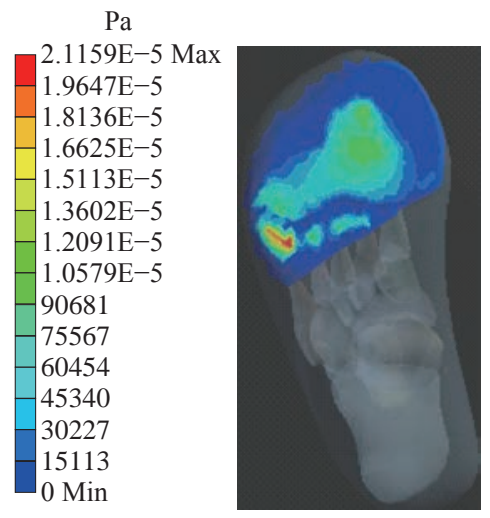

(d)

Fig. 3 Changes of plantar pressure distribution due to ligament injury in toes-off phase: (a) Talofibulare anterius ligament half injury; (b) talofibulare anterius ligament complete injury; (c) calcaneofibulare ligament half injury; and (d) calcaneofibulare ligament complete injury.

shows that as the injury degree of talofibulare anterius ligament and calcaneofibulare ligament deepened, the position of COP shifted more laterally. In the toes-off phase, the pulley structure made of tibia, fibula and talus entered joint capsule; the ankle joint was able to shift lateral and made itself unstable and vulnerable to sprain.

The stiffness of springs simulating talofibulare posterius ligament and calcaneofibulare ligament was set to half of the normal stiffness in Fig. 4(a) and (c), and to zero in Fig. 4(b) and (d), respectively, to simulate the injury of the ligaments. In the heelstike gait phase, talofibulare anterius ligament was completely injured, and therefore it could be ignored. Fig. 4 shows that as the injury degree of talofibulare posterius ligament and calcaneofibulare ligament deepened, lateral pressure under the tibio increased. However, in the heel-strike phase, the pulley structure made of tibia, fibula and talus was stable, and the contact area of these three bones was big, making the sprain less likely. Fig. 4 also shows that although the lateral pressure under the tibio increased, the extent of COP lateral shift increase was limited, which means the impact of ligament injury in the heel-strike phase is smaller than the toes-off phase.

Fig. 4 also shows that although the lateral pressure under the tibio increased, the extent of COP lateral shift increase was limited, which means the impact of ligament injury in the heel-strike phase is smaller than the toes-off phase.

Fig. 5 presents normalized COP displacements towards lateral and anaterolateral. The stiffness of talofibulare anterius ligament and calcaneofiulare ligament was reduced by $10 \%$ each time to obtain the contact pressure distribution. And then the figure of result was imported into MATLAB 2016 to calculate the coordinates of COP. Fig. 5 shows the accordance with the result of Mettler et al. that COP trajectory of CAI patients was more concentrated in the anterolateral region under the foot [16]. The injuries of lateral ligaments in toes-off phase were graded according to the change of slope as shown in Fig. 5. The grading result is shown in Table 2.

Fig. 6 presents the relation between the injury of talofibulare posterius ligament and calcaneofibulare 


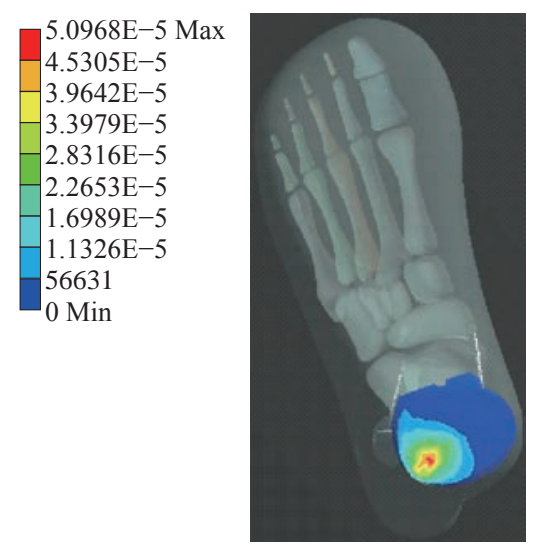

(a)

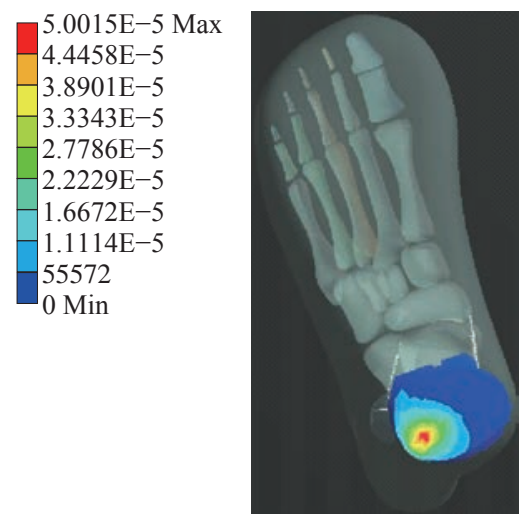

(c)

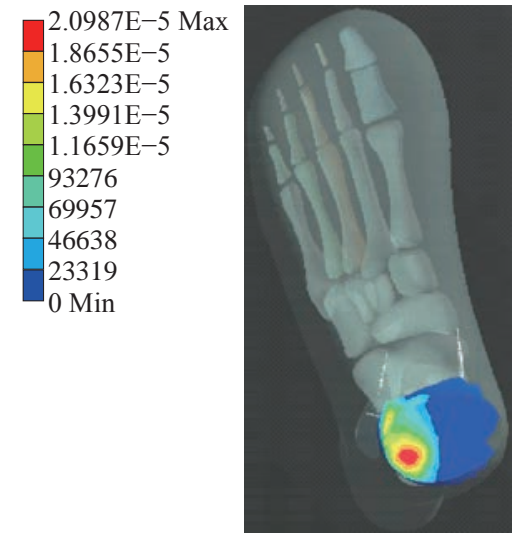

(b)

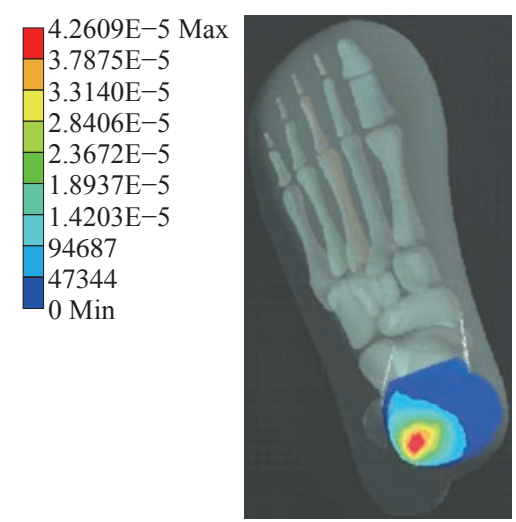

(d)

Fig. 4 Changes of plantar pressure distribution due to ligament injury in heel-strike phase: (a) Talofibulare posterius ligament half injury; (b) talofibulare posterius ligament complete injury; (c) calcaneofibulare ligament half injury; and (d) calcaneofibulare ligament complete injury.
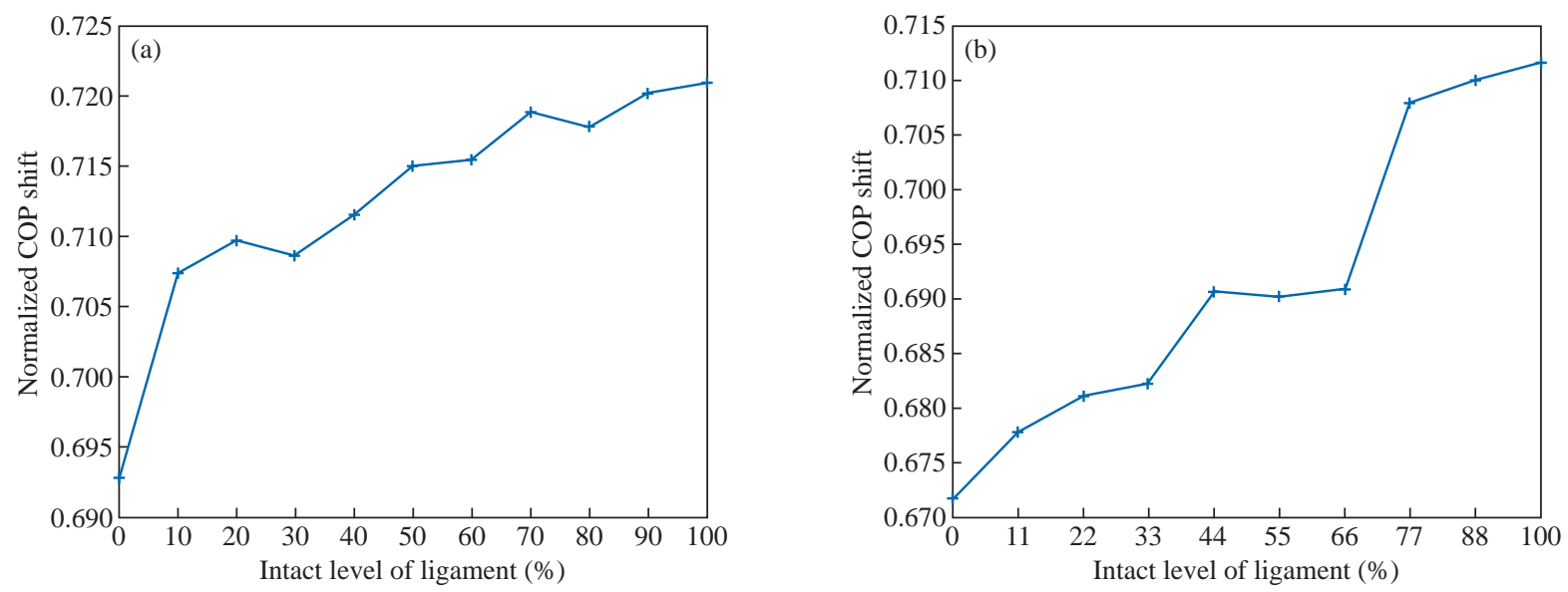

Fig. 5 Relation between injuries of talofibulare anterius ligament and calcaneofiulare ligament with COP shift in the toes-off phase: (a) Talofibulare anterius ligament; and (b) calcaneofiulare ligament.

Table 2 Grading lateral ligament laxity with COP shift in the toes-off phase

\begin{tabular}{cccc}
\hline & Slight & Medium & Severe \\
\hline Alofibulare anterius ligament & $0-40 \%$ & $40 \%-90 \%$ & $90 \%-100 \%$ \\
Calcaneofiulare ligament & $0-56 \%$ & $56 \%-78 \%$ & $78 \%-100 \%$ \\
\hline
\end{tabular}

ligament and the anteriolateral shift of COP coordinates in the heel-strike gait phase. The more injury of these two ligaments, the more shift COP laterally concentrated. It also shows that if the injury of ligaments were within certain extent, the shift of COP coordinates came into a plateau.

The injury of lateral ligaments in heel strike phase were graded according to the change of slope as shown 

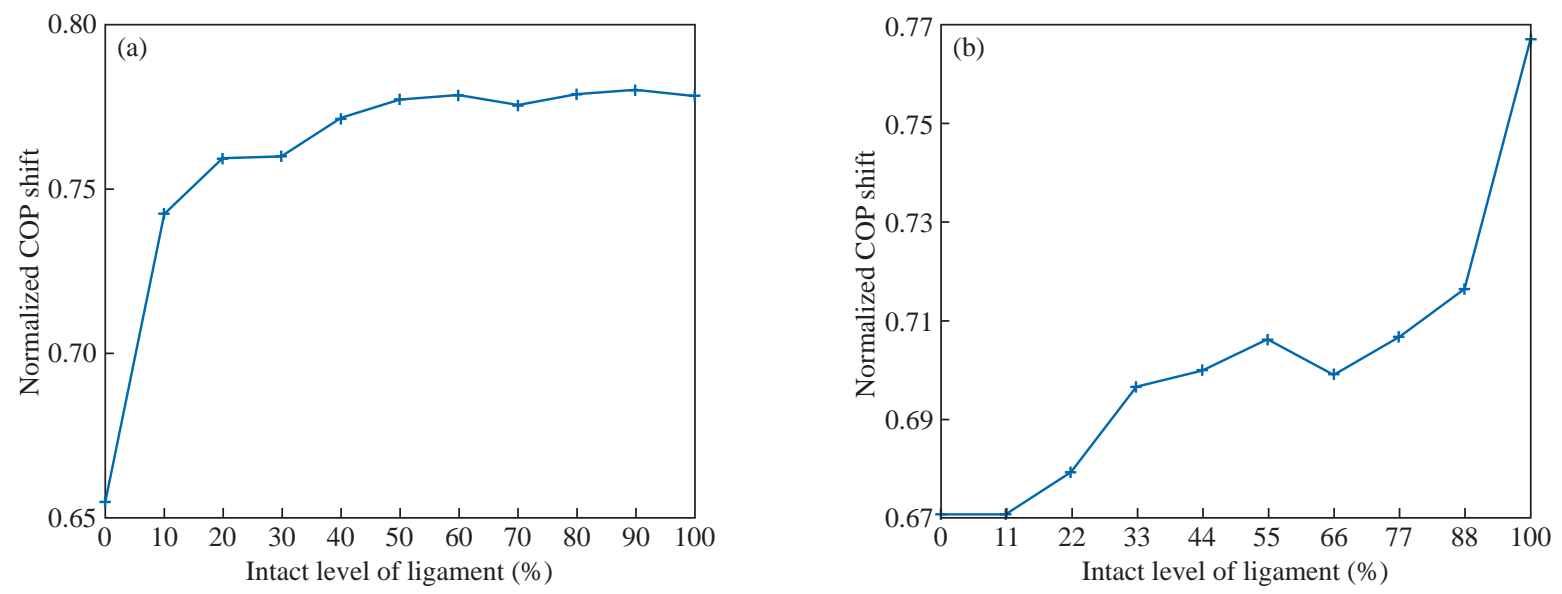

Fig. 6 Relation between injuries of talofibulare posterius ligament calcaneofibulare ligament with COP shift in the heel strike phase: (a) Talofibulare posterius ligament; and (b) calcaneofibulare ligament.

Table 3 Grading lateral ligament laxity with COP shift in the heel-strike phase

\begin{tabular}{cccc}
\hline & Slight & Medium & Severe \\
\hline Talofibulare posterius ligament & $0-10 \%$ & $10 \%-90 \%$ & $90 \%-100 \%$ \\
Calcaneofibulare ligament & $0-30 \%$ & $30 \%-60 \%$ & $60 \%-100 \%$ \\
\hline
\end{tabular}

in Fig. 6. The grading result is shown in Table 3.

It is also worth pointing out that the injury of lateral ligaments have different effects on the shift of COP coordinates. Posterior talofibulare ligament only affects COP coordinates during the heel-strike phase and anterior talofibulare ligament only affects COP coordinates during the toes-off phase, while calcaneofibulare ligament affects COP coordinates in the whole support phase. Although anterior talofibulare ligament and calcaneofibulare ligaments both affect in the toes-off phase, yet they have different extent of effects. Therefore, it may be able to distinguish the damage of anterior talofibulare ligament from that of calcaneofibulare ligament.

The stiffness of springs that stimulate lateral ligaments in neutral stance phase was set to $100 \%$ and $0 \%$ of the normal value in Fig. 7(a) and (b) respectively. The shift ratios of COP coordinates change were about $10 \%$ and $6 \%$ when the intact lateral ligament became completely injured respectively in heel-strike phase and toes-off phase, while that change in the neural phase was only $3 \%$. It indicates heelstrike phase and toes-off phase need to be paid more attention when studying the COP parameters of the patients with foot and ankle lesion.

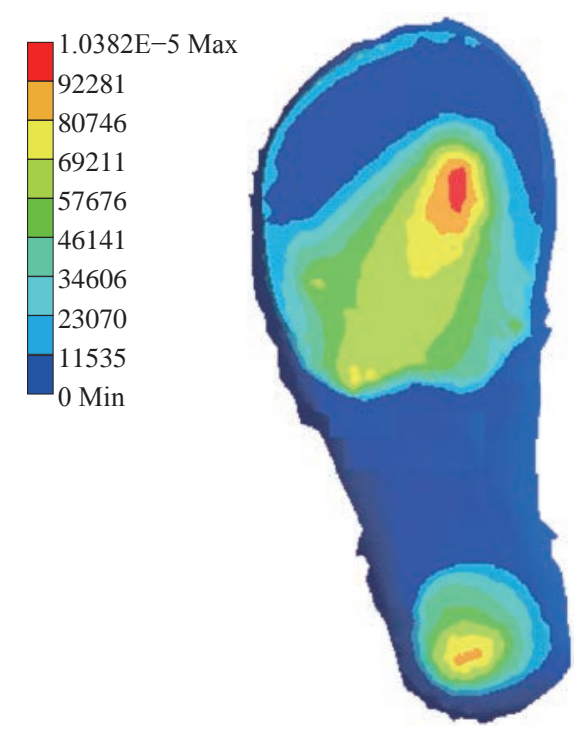

(b)

Fig. 7 Changes of plantar pressure distribution due to ligament injury in neutral stance phase: (a) Intact lateral ligaments; and (b) completely broken lateral ligametns. 


\section{Conclusions}

In this study, finite element models of toes-off and heel-strike gait phases were built via adjusting the corresponding joint ankle of existing neutral foot-ankle model according to the natural gait metrics. The plantar pressure distribution of MAI patients was studied via simulating the injury of lateral ligaments with the springs in ANSYS. COP of MAI patients shifts anterolateral and can be used to grade the injury degree of their lateral ligaments, which provides a possibility for monitoring the ligaments injury and rehabilitation progress of CAI patients quantitatively. The injury degree of talofibulare anterius ligament can be divided into three levels: $0-40 \%, 40 \%-90 \%$ and $90 \%-100 \%$. The injury degree of talofibulare posterius ligament can be divided into three levels: $0-10 \%, 10 \%-90 \%$ and $90 \%-100 \%$. The injury degree of calcaneofibulare ligament can be divided into three levels: $0-40 \%$, $40 \%-70 \%$ and $70 \%-100 \%$. It also validates the effectiveness of using simplified foot and ankle model to study the disease characterization.

\section{Conflict of Interests}

The authors declare that no competing interest exists.

\section{References}

[1] N. Maffulli, N.A. Ferran, Management of acute and chronic ankle instability. J Am Acad Orthop Surg, 2008, 16(10): 608-615.

[2] R.K. Parasher, D.R. Nagy, A.L. Em, et al., Clinical measurement of mechanical ankle instability. Manual Therapy, 2012, 17: 470-473

[3] F. Corazza, A. Leardini, J.J. O'Connor, et al., Mechanics of the anterior drawer test at the ankle: the effects of ligament viscoelasticity. Journal of Biomechanics, 2005, 38: 2118-2123.

[4] M. Guelfi, M. Zaperetti, A. Pantalone,et al., Open and arthroscopic lateral ligament repair for treatment of chronic ankle instability: A systematic review, 2018, 24:
11-18

[5] N.L.W. Keijsers, N.M. Stolwijk, J.W.K. Louwerens, et al., Classification of forefoot pain based on plantar pressure measurements. Clinical Biomechanics, 2013, 28: 350356.

[6] K.J. Mickle, B.J. Munro, S.R. Lord, et al., Gait, balance and plantar pressures in older people with toe deformities. Gait and Posture, 2011, 34: 347-351.

[7] W. Niu, T. Tang, M. Zhang, et al., An in vitro and finite element study of load redistribution in the midfoot. Life Sciences, 2014, 12: 1191-1196.

[8] J. Yu, J.T.M. Cheung, Y. Fan, et al., Development of a finite element model of female foot for high-heeled shoe design. Clinical Biomechanics, 2008, 23: 31-38.

[9] J.T.M. Cheung, M. Zhang, A.K.L. Leung, et al., Threedimensional finite element analysis of the foot during standing - a material sensitivity study. Journal of Biomechanics, 2005, 38: 1045-1054.

[10] R. Krishnan, M. Kopacz, and G.A. Ateshian, Experimental verification of the role of interstitial fluid pressurization in cartlilage lubrication. J Orthop Res, 2004, 22(3): 565-570.

[11] J.T.M. Cheung, M. Zhang, 2005. A 3-dimensional finite element model of the human foot and ankle for insole design. Arch. Phys. Med. Rehabil, 2004, 86: 353-358.

[12] J.T.M. Cheung, M. Zhang, Finite element modeling of the human foot and footwear. Proceedings of ABAQUS Users' Conference. 2006: 145-159.

[13] B. Nie, M.B. Panzer, A. Mane, et al., Determination of the in situ mechanical behavior of ankle ligaments. Journal of the Mechanical Behavior of Biomedical Materials, 2017, 65: 502-512.

[14] K. Tao, D. Wang, C. Wang, et al., An in vivo experimental validation of a computational model of human foot. Journal of Bionic Engineering, 2009, 6(4): 387-397.

[15] M. Nyska, S. Shabat, A. Simkin, et al., Dynamic force distribution during level walking under the feet of patients with chronic ankle instability. Br J Sports Med, 2003, 37(6): 495-497.

[16] K.E. Morrison, D.J. Hudson, I.S. Davis, et al., Plantar pressure during running in subjects with chronic ankle instability. Foot Ankle Int, 2010, 31(11): 994-1000.

[17] A. Mettler, L. Chinn, S.A. Saliba, et al., Balance training and center-of-pressure location in participants with chronic ankle instability. $J$ Athl Train, 2015, 50(4): 343349.

[18] Z. Miodonska, P. Stepien, P. Badura, et al., Inertial databased gait metrics correspondence to Tinetti Test and Berg Balance Scale assessments. Biomedical Signal Processing and Control, 2018, 7(44): 38-47.

Copyright $\subseteq$ Dian Wang, Ping Cai. This is an open-access article distributed under the terms of the Creative Commons Attribution License, which permits unrestricted use, distribution, and reproduction in any medium, provided the original author and source are credited. 